\title{
Which dialysis unit blood pressure is the most accurate for predicting home blood pressure in patients undergoing hemodialysis?
}

\author{
In-Cheol Yoon, Hye-Min Choi, and Dong-Jin Oh
}

Department of Internal Medicine, Myongji Hospital, Seonam University College of Medicine, Goyang, Korea

Received: August 16, 2015

Revised : September 25, 2015

Accepted: October 3, 2015

\section{Correspondence to}

Dong-Jin Oh, M.D.

Department of Internal

Medicine, Myongji Hospital,

Seonam University College of

Medicine, 55 Hwasu-ro 14beon-gil,

Deokyang-gu, Goyang 10475, Korea

Tel: $+82-31-810-5420$

Fax: $+82-31-969-0500$

E-mail: intmdoh@hanmail.net
Background/Aims: We investigated which dialysis unit blood pressure (BP) is the most useful for predicting home BP in patients undergoing hemodialysis (HD). Methods: Patients undergoing HD who had been treated $>3$ months were included in this study. Exclusion criteria were hospitalized patients with acute illness and changes in dry weight and anti-hypertensive drugs 2 weeks before the study. We used the dialysis unit BP recording data, such as pre-HD, intra-HD, post-HD, mean pre-HD, and post-HD (pre-post-HD), mean pre-HD, intra-HD, and postHD (pre-intra-post-HD) BP. Home BP (the same period of dialysis unit BP) was monitored as a reference method during 2 weeks using the same automatic oscillometric device. Patients were asked to record their BP three times daily (wake up, between noon and 6:00 PM, and at bedtime).

Results: Significant differences were detected between home systolic blood pressure (SBP) and pre-HD, post-HD, and intra-HD SBP $(p=0.003, p=0.001, p=0.016$, respectively). In contrast, no differences were observed between home SBP and pre-intra-post-HD and pre-post-HD SBP $(p=0.235, p=0.307$, respectively). Areas under the receiver operating characteristic curve for pre-intra-post-HD and prepost-HD SBP with 2-week home BP as the reference standard were 0.812 and 0.801 , respectively.

Conclusions: These results suggest that pre-intra-post-HD and pre-post-HD SBP had similar accuracy for predicting mean 2-week home SBP in HD patients. Therefore, pre-intra-post-HD and pre-post-HD SBP should be useful for predicting home SBP in HD patients if ambulatory or home BP measurements are unavailable.

Keywords: Renal dialysis; Blood pressure; Hypertension; Home; Hemodialysis units, hospital

\section{INTRODUCTION}

Mortality rates of patients undergoing hemodialysis (HD) are $20 \%$ during the first year of treatment and $70 \%$ after 5 years [1]. Cardiovascular disease is responsible for $>50 \%$ of deaths $[1,2]$. Only $20 \%$ of patients undergoing
HD are normotensive without the use of anti-hypertensive medication. Of those who are hypertension (HT), only $30 \%$ are controlled $[3,4]$. In addition, white-coat HT and masked HT were prevalent in Korean hypertensive population and there was a tendency of mean arterial pressure reduction in Korean dialysis populations $[5,6]$. 
Therefore, a unique challenge in patients undergoing $\mathrm{HD}$ is how and when blood pressure (BP) should be measured.

Ambulatory BP is best correlated with echocardiographic left ventricular hypertrophy and all-cause mortality compared to dialysis unit BP measurements $[7,8]$. However, ambulatory BP is not easily measured in a clinical setting. Emerging data suggest that home blood pressure (HBP) measurements are valid and useful to diagnose $\mathrm{HT}$ in patients undergoing $\mathrm{HD}$ [9]. In addition, HBP is as good as ambulatory BP for predicting target organ damage as assessed by echocardiographic left ventricular hypertrophy [7]. Finally, HBP is cheaper, easier, and more reproducible than those of ambulatory $\mathrm{BP}$, as it eliminates the white-coat effect and masked HT [10]. Therefore, ambulatory BP can be replaced by HBP for managing HT in patients undergoing HD. HBP measurements require patient compliance and equipment education [10]. Thus, HBP cannot be easily or precisely obtained, particularly in patients undergoing HD with low compliance and low educational status.

Dialysis unit BP can be measured very easily and routinely, although variations in the measurements have raised questions about its clinical usefulness [11]. It is unreasonable for nephrologists to discard dialysis unit BP measurements when managing HT in patients undergoing HD. In addition, the relationship between HBP and dialysis unit BP measurements has not been established. Thus, we investigated which dialysis unit $\mathrm{BP}$ measurement is the most useful for predicting HBP in patients undergoing HD.

\section{METHODS}

\section{Subjects}

Patients were selected from a single dialysis unit located in Goyang, Korea. Inclusion criteria were men or women aged $\geq 20$ years who had chronic kidney disease of any etiology, were undergoing HD three times/week for at least 3 months; hemoglobin $\geq 10$ and $\leq 12 \mathrm{~g} / \mathrm{dL}$; single-poll Kt/V $\geq 1.2$, and serum albumin $\geq 3.5 \mathrm{~g} / \mathrm{dL}$. Exclusion criteria were patients who had visual or cognitive insufficiency, cardiac arrhythmias, severe heart or liver failure, and pregnancy. Those who missed two or more HD treatments over 1 month, had chronic atrial fibril- lation, body mass index $\geq 30 \mathrm{~kg} / \mathrm{m}^{2}$, had a change in dry weight or anti-hypertensive medication 2 weeks before the study, or had a single episode of intradialytic hypotension (systolic blood pressure $[\mathrm{SBP}]<90 \mathrm{mmHg}$ ) were also excluded. HT was defined as mean pre-DBP from six consecutive sessions $\geq 140 \mathrm{mmHg}$ SBP and/or $\geq 90$ $\mathrm{mmHg}$ diastolic blood pressure (DBP) and/or use of anti-hypertensive medication. Medications and dry weight (the post-HD weight without HT, intra-dialytic hypotension, pulmonary congestion and peripheral edema) were held constant during our study. Informed written consent was obtained from all patients. This study was approved by the local research Myongin Hospital Institutional Review Board.

\section{Dialysis technique}

All patients underwent high-flux HD three times weekly with bicarbonate buffer for 4 hours via left forearm arteriovenous fistula, using 1.0 to $1.4 \mathrm{~m}^{2}$ hollow fiber polysulfone membranes, blood flow rates of 250 to 280 $\mathrm{mL} / \mathrm{min}$, and a dialysate flow rate of $500 \mathrm{~mL} / \mathrm{min}$, using the Fresenius 5008S machine (Fresenius Medical Care, Bad Homburg, Germany). UF volume was 1,500 to 3,000 $\mathrm{mL}$, and treatment time was 4 hours. The dialysis fluid contained $138 \mathrm{mEq} / \mathrm{L}$ sodium, $2 \mathrm{mEq} / \mathrm{L}$ potassium, $2.5 \mathrm{mEq} / \mathrm{L}$ calcium, $1.0 \mathrm{mEq} / \mathrm{L}$ magnesium, $108.5 \mathrm{mEq} / \mathrm{L}$ chloride, $35 \mathrm{mEq} / \mathrm{L}$ bicarbonate, and $99.1 \mathrm{mg} / \mathrm{dL}$ glucose, and temperature was maintained at $36^{\circ} \mathrm{C}$.

\section{Home blood pressure monitoring}

HBP monitoring was performed using the HBP measuring devices (Omron HEM model 705-CP automatic oscillometric device, Omron Healthcare, Kyoto, Japan). The patients were trained on use of the device and instructed to measure their BP three times daily (wake up, between noon and 6:00 PM, and at bedtime) three consecutive times on each occasion for 2 weeks (the same period of dialysis unit BP). The recordings were accepted for interpretation if the patient had at least $18 \mathrm{BP}$ measurements during 1 week.

\section{Dialysis unit blood pressure}

$\mathrm{BP}$ at the dialysis unit before each dialysis session (preHD BP) was measured by nursing staff using the same HBP measuring devices (Omron HEM model 705-CP automatic oscillometric device). BP was recorded at 1-, 
2-, 3-hour and immediately after HD. The mean of the 1-, 2-, and 3-hour BPs was considered the intra-HD BP. BP measured immediately after dialysis was post-HD BP. Three readings at each visit were averaged to provide one value. These $\mathrm{BP}$ measurements were averaged for 2 weeks (the same period of HBP monitoring). Thus, each patient had six pre-HD, six post-HD, and six intra-HD BP recordings for dialysis unit BP.

\section{Index and reference blood pressure}

The following methods were used to define summary measures for various $\mathrm{BP}$ recordings:

Pre-HD BP: SBP measurements obtained before six HD treatments were averaged. The DBP measurements obtained before HD were averaged separately over these six treatments.

Post-HD BP: SBP measurements obtained immediately after six HD treatments were averaged. DBP measurements obtained separately after HD were averaged over these six treatments.

Intra-HD BP: SBP measurements obtained during a single treatment were averaged. These averages from a single HD treatment were averaged over six treatments to yield a single intra-HD SBP. DBP averages were calculated analogously.

Intra-HD BP, including pre-HD and post-HD BP: These measurements were obtained as for intra-HD BP, except that the pre-HD and post-HD BP measurements were also included before averaging the measurements (expressed as mean pre-intra-post-HD BP).

Pre-HD and post-HD BP: SBP values obtained before and immediately after dialysis were averaged. These averages were averaged to yield mean pre-HD and postHD BP over six treatments (expressed as mean pre-postHD BP).

HBP average: 2-week HBP values were averaged to yield the overall $\mathrm{HBP}$ value as the reference $\mathrm{BP}$.

Home systolic blood pressure (HSBP) $\geq 150 \mathrm{mmHg}$ was used to classify patients with systolic home HT. The threshold of $\geq 150 \mathrm{mmHg}$ from the HBP measurement was derived from Agarwal et al. [11] who compared HBP to ambulatory BP measurements in patients undergoing $\mathrm{HD}$ and demonstrated that $\mathrm{HSBP} \geq 150 \mathrm{mmHg}$ averaged over 1 week had the best combination of sensitivity (80\%) and specificity (84.1\%).

\section{Measurement of agreement}

The mean difference (MD) and the limits of agreement between HSBP and pre-HD, post-HD, intra-HD, preintra-post-HD SBP, and pre-post-HD SBP were calculated using Bland-Altman plots.

\section{Measurement of diagnostic test performance}

We calculated the area under the receiver operating characteristics (ROC) curve and their 95\% confidence intervals (CIs) for pre-HD, intra-HD, post-HD, pre-intra-post-HD, and pre-post-HD SBP with 2-week HSBP.

\section{Statistical analysis}

Variables are expressed as mean \pm standard deviation (SDs). We utilized the unpaired $t$ test and repeated measures analysis of variance to compare HBP with dialysis unit BP. The MDs between HSBP and pre-HD, post$\mathrm{HD}$, intra-HD, pre-intra-post-HD SBP, and pre-postHD SBP were assessed for significance using the one sample $t$ test. A $p<0.05$ was considered significant.

\section{RESULTS}

\section{Sample characteristics and mean blood pressure}

Seventy HD patients in our clinic were recruited. The study flow is outlined in Fig. 1. Thirty-five patients had adequate data, and their clinical characteristics are summarized in Table 1. Mean HBP (systolic and diastolic) were $144.3 \pm 12.9$ and $74.5 \pm 14.0 \mathrm{mmHg}$, respectively. Mean pre-HD BP values were $152.9 \pm 16.9$ and $70.1 \pm 13.6$

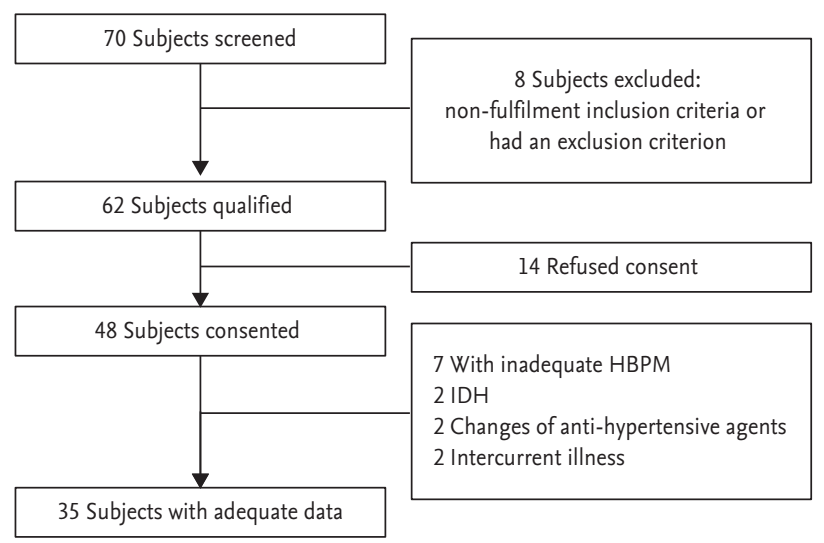

Figure 1. Study flow diagram. HBPM, home blood pressure monitoring; IDH, intra-dialytic hypotension. 
Table 1. Baseline characteristics of the study population $(\mathbf{n}=35)$

\begin{tabular}{lc}
\hline Clinical characteristic & Value \\
\hline Age, yr & $60 \pm 14.4$ \\
Male sex & $20(57)$ \\
Predialysis weight, kg & $61.8 \pm 11.5$ \\
Postdialysis weight, kg & $59.5 \pm 11.0$ \\
Interdialytic weight gain, kg & $2.0 \pm 1.0$ \\
Body mass index, kg/m ${ }^{2}$ & $21.7 \pm 4.1$ \\
Hemodialysis, yr & $3.4 \pm 3.0$ \\
Etiology of end-stage renal disease & \\
\hline Diabetes mellitus & $15(43)$ \\
Hypertension & $12(34)$ \\
Glomerulonephritis & $7(20)$ \\
Other & $1(3)$ \\
Kt/Vurea & $1.5 \pm 0.3$ \\
Albumin, g/dL & $3.8 \pm 0.4$ \\
Hemoglobin, g/dL & $11.2 \pm 1.8$ \\
No. receiving antihypertensive drugs & $19(54)$ \\
No. of antihypertensives in users & $2.3 \pm 1.2$ \\
2-Week home blood pressure, mmHg & $144.3 \pm 12.9 /$ \\
& $74.5 \pm 14.0$ \\
\hline Values are presented as mean \pm SD $0 r(\%)$
\end{tabular}

Values are presented as mean $\pm \mathrm{SD}$ or number (\%).

Table 2. Comparison of home blood pressure and dialysis unit blood pressure measurements

\begin{tabular}{|c|c|c|}
\hline Variable & SBP & DBP \\
\hline Home & $144.3 \pm 12.9$ & $74.5 \pm 14.0$ \\
\hline \multicolumn{3}{|l|}{ Dialysis unit } \\
\hline Pre-HD & $152.9 \pm 16.9^{\mathrm{a}}$ & $70.1 \pm 13.6^{\mathrm{a}}$ \\
\hline Post-HD & $133.8 \pm 19.6^{\mathrm{a}}$ & $68.6 \pm 13.9^{\mathrm{a}}$ \\
\hline Intra-HD (1 hr-3 hr) & $137.7 \pm 17.0^{a}$ & $68.1 \pm 14.0^{\mathrm{a}}$ \\
\hline $1 \mathrm{hr}$ & $139.7 \pm 18.4^{a}$ & $68.3 \pm 14.7^{\mathrm{a}}$ \\
\hline $2 \mathrm{hr}$ & $138.1 \pm 19.8^{a}$ & $68.0 \pm 14.5^{\mathrm{a}}$ \\
\hline $3 \mathrm{hr}$ & $135.6 \pm 18.2^{\mathrm{a}}$ & $68.5 \pm 14.5^{\mathrm{a}}$ \\
\hline Pre-intra-post-HD & $142.3 \pm 16.4$ & $68.3 \pm 13.7^{\mathrm{a}}$ \\
\hline Pre-post-HD & $143.4 \pm 15.1$ & $69.4 \pm 12.4^{\mathrm{a}}$ \\
\hline
\end{tabular}

Values are presented as mean $\pm \mathrm{SD}$.

SBP, systolic blood pressure; DBP, diastolic blood pressure; Home, home SBP and DBP; HD, hemodialysis; pre-HD, mean pre-dialysis SBP and DBP; post-HD, mean post-dialysis SBP and DBP; intra-HD ( $1 \mathrm{hr}-3 \mathrm{hr})$, mean intra-dialysis (1 hr-3 hr) SBP and DBP; pre-intra-post-HD, mean pre- and intra- and post-dialysis SBP and DBP; pre-post-HD, mean pre- and post-dialysis SBP and DBP.

${ }^{a} p<0.05$ vs. home blood pressure.
$\mathrm{mmHg}$, respectively. Mean post-HD BP values were $133.8 \pm 19.6$ and $68.6 \pm 13.9 \mathrm{mmHg}$, respectively. Mean intra-HD (1- to 3-hour) BP values were $137.7 \pm 17.0$ and $68.1 \pm 14.0 \mathrm{mmHg}$, respectively. Mean intra-HD (1-hour) BP, $139.7 \pm 18.4,68.3 \pm 14.7 \mathrm{mmHg}$; mean intra-HD (2hour) BP, $138.1 \pm 19.8,68.0 \pm 14.5 \mathrm{mmHg}$; mean intra-HD (3-hour) BP, 135.6 $\pm 18.2,68.5 \pm 14.5 \mathrm{mmHg}$. Comparisons showed significant differences between HBP and mean pre-HD, post-HD, and intra-HD (1- to 3-hour) BP, intra-HD (1-hour) BP, intra-HD (2-hour) BP, intra-HD (3hour) BP, regardless of SBP and DBP $(p<0.05)$ (Table 2). Mean pre-intra-post-HD BP values were $142.3 \pm 16.4$ and $68.3 \pm 13.7 \mathrm{mmHg}$, respectively. Mean pre-post-HD SBP and DBP values were $143.4 \pm 15.1$ and $69.4 \pm 12.4 \mathrm{mmHg}$, respectively. No significant differences were observed between HBP and mean pre-intra-post-HD and prepost-HD BP, regardless of SBP and DBP (Table 2).

\section{Bland-Altman plot agreement analysis}

The Bland-Altman analysis is shown in Fig. 2. Mean BP is plotted against the difference, and the MD and the CI for the agreement $(\mathrm{MD} \pm 1.96 \mathrm{SD})$ were calculated.

The MD between pre-HD SBP and HSBP was -8.6 $\mathrm{mm} \mathrm{Hg}$. The CI of agreement between pre-HD SBP and HSBP was 22.6 (upper) and -39.8 (lower) $\mathrm{mmHg}$. The MD between post-HD SBP and HSBP was $10.5 \mathrm{mmHg}$. The CI of agreement between post-HD SBP and HSBP was 45.3 (upper) and -24.3 (lower) $\mathrm{mmHg}$. The MD between intra-HD SBP and HSBP was $6.7 \mathrm{mmHg}$. The CI of agreement between intra-HD SBP and HSBP was 37.4 (upper) and -24.0 (lower) mmHg. Comparisons showed significant differences between HSBP and pre-HD SBP, post-HD SBP, and intra-HD SBP $(p=0.003, p=0.001, p=$ 0.016 , respectively) (Fig. 2A-2C).

The MD between pre-intra-post-HD SBP and HSBP was $1.3 \mathrm{mmHg}$. The CI of agreement between pre-postHD SBP and HSBP was 14.4 (upper) and -11.8 (lower) mmHg. The MD between pre-post-HD SBP and HSBP was $1.2 \mathrm{mmHg}$. The CI of agreement between pre-postHD SBP and HSBP was 15.1 (upper) and -12.7 (lower) mmHg. Comparisons showed no differences between HSBP and pre-intra-post-HD SBP or pre-post-HD SBP $(p=0.235, p=0.307$, respectively) (Fig. $2 \mathrm{D}$ and $2 \mathrm{E})$. 

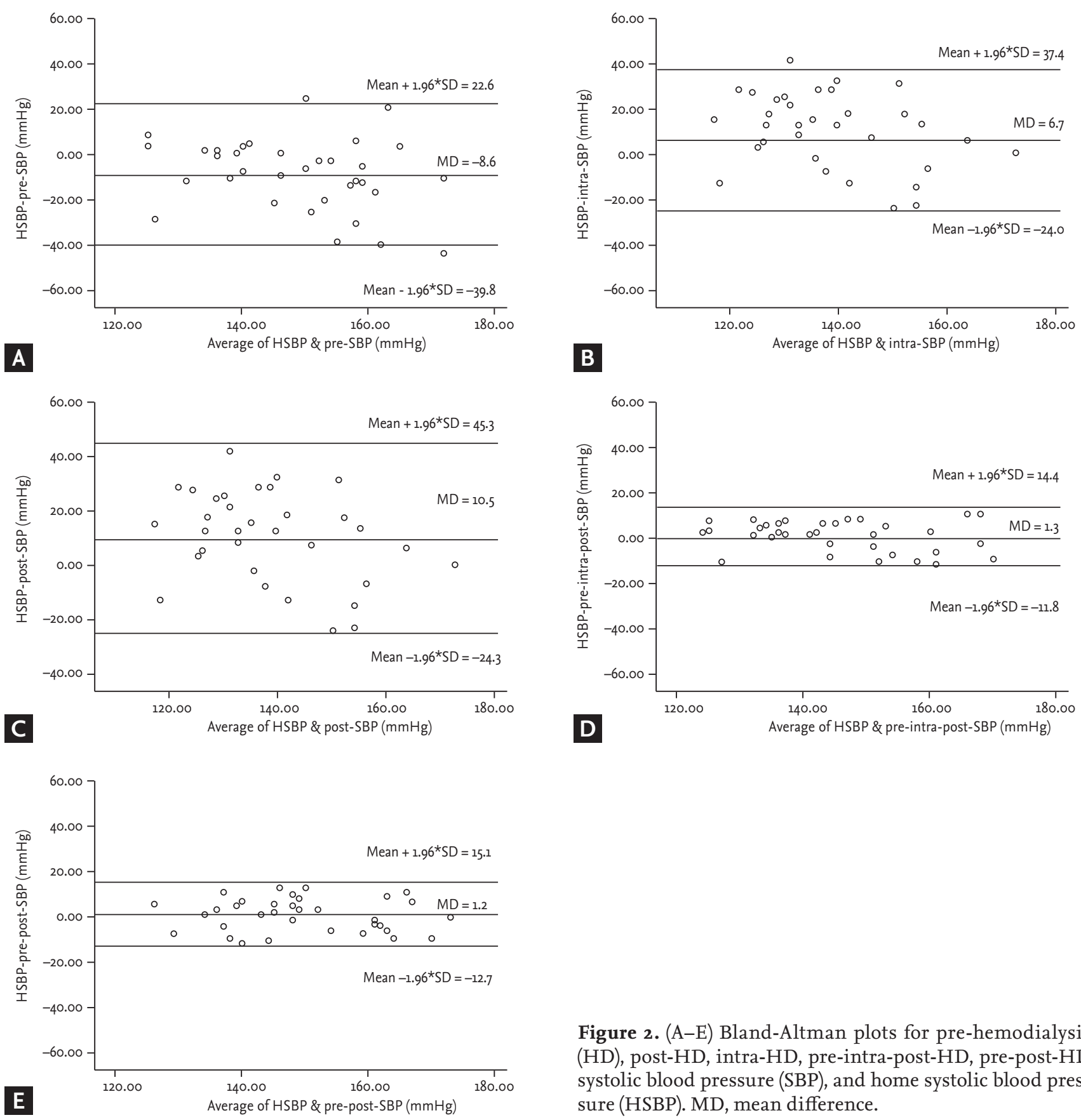

Figure 2. (A-E) Bland-Altman plots for pre-hemodialysis (HD), post-HD, intra-HD, pre-intra-post-HD, pre-post-HD systolic blood pressure (SBP), and home systolic blood pressure (HSBP). MD, mean difference.

Diagnostic performance of mean pre-HD, post-HD, intra-HD, pre-intra-post-HD, pre-post-HD SBP with 2-week HBP as the reference standard

The areas under the ROC curve (95\% CI) for pre-HD, post-HD, intra-HD, pre-intra-post-HD, and pre-postHD SBP were 0.745 (0.541 to 0.880 ), 0.701 (0.514 to 0.835 ), 0.738 ( 0.533 to 0.862 ), 0.812 ( 0.651 to 0.964 ), and 0.801 (0.643 to 0.960 ), respectively (Table 3). Fig. 3 shows the

ROC curves for pre-intra-post-HD, pre-post-HD SBP with 2-week HBP as the reference standard.

\section{DISCUSSION}

The major finding of our study was that mean pre-postHD and mean pre-intra-post-HD SBP had similar ac- 
Table 3. Area under the ROC curve for pre-HD, post-HD, intra-HD, pre-intra-post-HD, and pre-post-HD SBP using 2-week home SBP measurements

\begin{tabular}{lccccc}
\hline & \multicolumn{4}{c}{ SBP } \\
\cline { 2 - 6 } & Pre-HD & Post-HD & Intra-HD & Pre-intra-post-HD & Pre-post-HD \\
\hline $\begin{array}{l}\text { Area under ROC curve } \\
(95 \% \mathrm{CI})\end{array}$ & 0.745 & 0.701 & 0.738 & 0.812 & 0.801 \\
\hline
\end{tabular}

ROC, receiver operating characteristic; HD, hemodialysis; SBP, systolic blood pressure; CI, confidence interval.

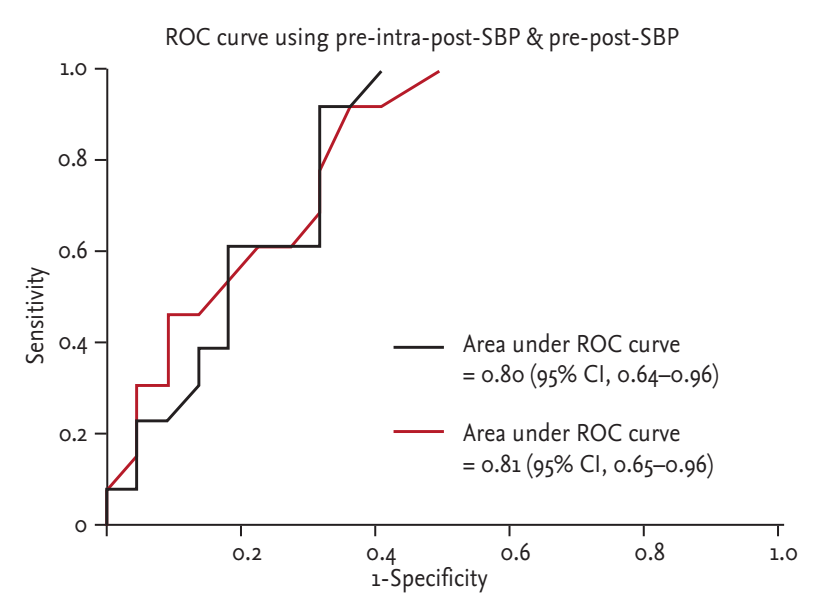

Figure 3. Area under the receiver operating characteristic (ROC) curve for pre-post-hemodialysis (HD) and pre-intrapost-HD systolic blood pressure using 2-week home blood pressure as the reference standard. CI, confidence interval.

curacy for predicting mean 2-week HSBP in patients undergoing HD.

The BP decrease during HD countervails the inter-HD increase in BP. Thus, a reciprocal relationship exists between the inter-HD increase in BP and the intra-HD fall in BP. These BP excursions within a short time period make applying traditional HT definitions problematic [12]. Given this variability, it is not surprising that pre-HD and post-HD measurements correlate only roughly with inter-HD ambulatory BP monitoring. A meta-analysis reporting on these variations indicated that predicting individual ambulatory $\mathrm{BP}$ using pre-HD or post-HD BP measurements could be erroneous by 35 $\mathrm{mmHg}$ in either direction [13]. In fact, a survey of UK centers showed that better post-dialysis BP targets had more intra-HD hypotension [14].

Agarwal et al. [15] concluded that considering intra-HD BP measurements together with pre-HD and post-HD BP measurements improves reproducibility, bias, precision, and accuracy of BP measurements compared to those of pre-HD or post-HD measurements using ambulatory BP as a reference method. Because averaging many intra-HD BP measurements provides an accurate estimate of arterial pressure over a range of volume states, it is possible that averaging many intra-HD recordings provides a better relationship with inter-HD HBP recordings. Unexpectedly, we found that mean pre-post-HD SBP had similar diagnostic ability compared to that of mean pre-intra-post-HD SBP measurements for predicting HSBP. We took hourly dialysis unit measurements. The mean pre-intra-post-HD SBP measurements were not as strong as the pre-post-HD SBP measurements because of the infrequent BP measurements in our study. Thus, it was unlikely that significant difference would be found between mean prepost-HD SBP and mean pre-intra-post-HD SBP for predicting HSBP using hourly intra-HD BP recordings. Hourly intra-HD BP recordings are performed in most Korean dialysis centers because of patient convenience and request.

Mitra et al. [16] reported that BP measured 20 minutes after dialysis (P2oBP) agrees best with inter-HD ambulatory BP. They suggested that inter-HD ambulatory BP significantly underestimates BP if BP is recorded immediately after HD. However, Batlle et al. [17] observed a sustained decline in BP up to 1 hour after HD. This discrepancy could be related to differences in baseline hydration of the study population. Therefore, it is questionable whether P2OBP is the best reliable post-HD BP for predicting inter-HD ambulatory BP. Waiting $>20$ minutes after HD to obtain post-HD BP is inconvenient and bothersome to almost all patients undergoing HD. Thus, we used BP immediately after HD as post-HD BP and $\mathrm{HBP}$ as the reference $\mathrm{BP}$, rather than ambulatory BP.

HBP is as good as ambulatory BP for predicting target organ damage as assessed by echocardiographic left ventricular hypertrophy [7]. Ambulatory BP can be replaced 
by HBP to manage HT in patients undergoing HD. However, HBP measurements require standardizing the device as well as patient compliance and education about the equipment [10]. In addition, HBP cannot be easily or precisely obtained in some clinical settings. Finally, Although Agarwal et al. [13] indicated that pre and post dialysis BP are imprecise estimates of interdialytic ambulatory $\mathrm{BP}$, our results showed that mean dialysis unit BP can be a useful predictor of HBP. Therefore, mean pre-intra-post-HD and mean pre-post-HD SBP might be more useful for predicting HSBP in patients undergoing HD with poor socioeconomic status.

This study had some limitations. First, the number of patients was relatively small and they were all from a single-center. Second, ambulatory BP monitoring should have been performed and compared to our data to strengthen our results. We did not assess reproducibility of ambulatory BP. However, this was not the purpose of the study. Third, we did not present the DBP data because it is controversial for defining home diastolic HT.

In conclusion, mean pre-post-HD and mean pre-intra-post-HD SBP should be very useful for predicting HSBP in patients undergoing HD if ambulatory or HBP measurements are unavailable. However, these readings should be used with caution with the understanding that they are inferior to ambulatory or HBP measurements.

\section{KEY MESSAGE}

1. Home blood pressure (HBP) is as good as ambulatory blood pressure (BP) for predicting target organ damage as assessed by echocardiographic left ventricular hypertrophy. Ambulatory BP can be replaced by HBP to manage hypertension (HT) in patients undergoing hemodialysis (HD).

2. Mean pre-post-HD and mean pre-intra-postHD systolic BP should be very useful for managing HT predicting home systolic BP in patients undergoing $\mathrm{HD}$ if ambulatory or $\mathrm{HBP}$ measurements are unavailable.

3. However, these readings (mean pre-post-HD and mean pre-intra-post-HD systolic BP) should be used with caution with the understanding that they are inferior to ambulatory or HBP measurements.

\section{Conflict of interest}

No potential conflict of interest relevant to this article was reported.

\section{REFERENCES}

1. Collins AJ, Kasiske B, Herzog C, et al. USRDS 2006 Annual Data Report. Am J Kidney Dis 2006; 47(Suppl 1):1-286.

2. Rayner HC, Pisoni RL, Bommer J, et al. Mortality and hospitalization in haemodialysis patients in five European countries: results from the Dialysis Outcomes and Practice Patterns Study (DOPPS). Nephrol Dial Transplant 2004;19:108-120.

3. Sarnak MJ, Levey AS, Schoolwerth AC, et al. Kidney disease as a risk factor for development of cardiovascular disease: a statement from the American Heart Association Councils on Kidney in Cardiovascular Disease, High Blood Pressure Research, Clinical Cardiology, and Epidemiology and Prevention. Hypertension 2003;42:1050-1065.

4. Agarwal R, Nissenson AR, Batlle D, Coyne DW, Trout JR, Warnock DG. Prevalence, treatment, and control of hypertension in chronic hemodialysis patients in the United States. Am J Med 2003;115:291-297.

5. Hwang ES, Choi KJ, Kang DH, et al. Prevalence, predictive factor, and clinical significance of white-coat hypertension and masked hypertension in Korean hypertensive patients. Korean J Intern Med 2007;22:256-262.

6. Jin DC. Major changes and improvements of dialysis therapy in Korea: review of end-stage renal disease registry. Korean J Intern Med 2015;30:17-22.

7. Agarwal R, Brim NJ, Mahenthiran J, Andersen MJ, Saha C. Out-of-hemodialysis-unit blood pressure is a superior determinant of left ventricular hypertrophy. Hypertension 2006;47:62-68.

8. Alborzi P, Patel N, Agarwal R. Home blood pressures are of greater prognostic value than hemodialysis unit recordings. Clin J Am Soc Nephrol 2007;2:1228-1234.

9. Agarwal R. Managing hypertension using home blood pressure monitoring among haemodialysis patients: a call to action. Nephrol Dial Transplant 2010;25:1766-1771.

10. Agarwal R. Blood pressure and mortality among hemodialysis patients. Hypertension 2010;55:762-768.

11. Agarwal R. The controversies of diagnosing and treating hypertension among hemodialysis patients. Semin Dial 2012;25:370-376. 
12. Agarwal R. Systolic hypertension in hemodialysis patients. Semin Dial 2003;16:208-213.

13. Agarwal R, Peixoto AJ, Santos SF, Zoccali C. Pre- and postdialysis blood pressures are imprecise estimates of interdialytic ambulatory blood pressure. Clin J Am Soc Nephrol 2006;1:389-398.

14. Davenport A, Cox C, Thuraisingham R. Achieving blood pressure targets during dialysis improves control but increases intradialytic hypotension. Kidney Int 2008;73:759764.
15. Agarwal R, Metiku T, Tegegne GG, et al. Diagnosing hypertension by intradialytic blood pressure recordings. Clin J Am Soc Nephrol 2008;3:1364-1372.

16. Mitra S, Chandna SM, Farrington K. What is hypertension in chronic haemodialysis? The role of interdialytic blood pressure monitoring. Nephrol Dial Transplant 1999;14:2915-2921.

17. Batlle DC, von Riotte A, Lang G. Delayed hypotensive response to dialysis in hypertensive patients with end-stage renal disease. Am J Nephrol 1986;6:14-20 